International Journal of Advanced Research in Engineering and Technology (IJARET)

Volume 10, Issue 4, July-August 2019, pp. 63-71, Article ID: IJARET_10_04_008

Available online at http://iaeme.com/Home/issue/IJARET? Volume $=10 \&$ Issue $=4$

ISSN Print: 0976-6480 and ISSN Online: 0976-6499

(C) IAEME Publication

\title{
A COMPARATIVE STUDY OF SIZE PARAMETERS EFFECTS IN MESHLESS METHOD LOCAL PETROV-GALERKIN (MLPG) AND LOCAL RADIAL POINT INTERPOLATION METHOD (LRPIM)
}

\author{
Ahmed Moussaoui and Touria Bouziane \\ Department of physics, Faculty of Sciences, Moulay Ismail University \\ B.P.11201 Meknes, Morocco.
}

\begin{abstract}
We use a Meshless local Petrov-Galerkin method (MLPG) to analyse an elastostatic problem deformation of a homogeneous rectangular plate of two-dimensional. First the formulations of method local Petrov-Galerkin (MLPG) and Local radial point interpolation method (LRPIM) are obtained. The aim of this article is to study the convergence and accuracy of these methods: MLPG and LRPIM. Finally a comparative study of numerical results obtained is made.
\end{abstract}

Keywords: Meshless Methods; MLPG and LRPIM; Radial basis function; support domain; rectangular plate; Linear Elasticity.

Cite this Article: Ahmed Moussaoui and Touria Bouziane, A Comparative Study of Size Parameters Effects in Meshless Method Local Petrov-Galerkin (Mlpg) and Local Radial Point Interpolation Method (Lrpim), International Journal of Advanced Research in Engineering and Technology, 10(4), 2019, pp. 63-71.

$\mathrm{http}: / /$ iaeme.com/Home/issue/IJARET?Volume $=10 \& \mathrm{Issue}=4$

\section{INTRODUCTION}

In recent years, many of meshless methods have been developed and achieved remarkable progress, such element free Galerkin (EFG) method [1], meshless local Petrov-Galerkin (MLPG) method [2-3] and the point interpolation method (PIM) [4-5]. The meshless methods are developed using Galerkin weak form and shape functions are built in only on a group of nodes arbitrarily distributed in a local support domain by means of interpolation. A global background cell structure is required to evaluate the integration in the Galerkin weak-form. There are two types of the point interpolation method (PIM) shape functions have been used so far with different forms of basic functions: polynomial basis functions and radial basis 
functions (RBFs) [5] that is named as radial point interpolation method (RPIM). In this paper, a 2D elastostatic problem is formulated by meshless local Petrov-Galerkin (MLPG) method based on the Galerkin weak form using locally supported shape functions and the various types are notified. In section 3 the numerical example is given and some important parameters of RPIM are investigated. The efficiency convergence and accuracy of local radial point interpolation method (LRPIM) and by comparison with the MLPG is studied in final section.

\section{RPIM SHAPE FUNCTIONS IN MESHLESS METHOD}

$\mathrm{u}^{\mathrm{h}}(\mathrm{x})$ is composed of two part: $\mathrm{P}_{\mathrm{j}}(\mathrm{x})$ Polynomial basis functions and $R_{i}(x)$ the radial basis functions

$$
\text { RBFs [6-7]: } \quad \mathrm{u}^{\mathrm{h}}(\mathrm{x})=\sum_{\mathrm{i}=1}^{\mathrm{n}} \mathrm{R}_{\mathrm{i}}(\mathrm{x}) \mathrm{a}_{\mathrm{i}}+\sum_{\mathrm{j}=1}^{\mathrm{m}} \mathrm{P}_{\mathrm{j}}(\mathrm{x}) \mathrm{b}_{\mathrm{j}}
$$

$\mathbf{n}$ is the number of field nodes in the local support domain and $\mathbf{m}$ is the number of polynomial terms. Radial basis is a function of distance

$$
\text { r: } r=\sqrt{\left(x-x_{i}\right)^{2}+\left(y-y_{i}\right)^{2}}
$$

The above equation (1) can be expressed in the matrix form [7].

$$
\mathbf{U}_{1}=\mathbf{R} \times \mathbf{a}+\mathbf{P} \times \mathbf{b}
$$

Where $U_{1}$ the vector of function values: $U_{1}=\left[u_{1}, u_{2}, u_{3}, \ldots, u_{n}\right]^{T} ; \mathbf{R}$ is the moment matrix of RBFs; $\mathbf{P}$ is the moment matrix of Polynomial basis function and $\mathbf{a}, \mathbf{b}$ are the values of unknowns coefficients (Radial and Polynomial). We note that, to obtain the unique solutions of eq. (2), the constraint conditions should be applied as follows [8]:

$$
\sum_{i=1}^{n} P_{i j}(x) a_{i}=0, j=1,2, \ldots, m
$$

By combining Eqs. $(3,4)$ yields a set of equations in the matrix form:

$$
\overline{\mathrm{U}}_{1}=\left[\begin{array}{l}
\mathrm{U}_{1} \\
0
\end{array}\right]=\left[\begin{array}{ll}
\mathrm{R} & \mathrm{P} \\
\mathrm{P}^{\mathrm{T}} & 0
\end{array}\right]\left[\begin{array}{l}
\mathrm{a} \\
\mathrm{b}
\end{array}\right]=\mathrm{Ga}_{0}
$$

The unknown's vector can be obtained by inversion of the matrix

$$
\mathbf{G}=\left[\begin{array}{cc}
\mathbf{R} & \mathbf{P} \\
\mathbf{P}^{\mathbf{T}} & 0
\end{array}\right]
$$

Substitution of the vector obtained by inversion of matrixG into eq.(1) leads to:

$$
\mathrm{u}^{\mathrm{h}}(\mathrm{x})=\sum_{\mathrm{i}=1}^{\mathrm{n}} \phi_{\mathrm{i}} \mathrm{u}_{\mathrm{i}}
$$

\section{LOCAL WEAK FORM METHOD LRPIM}

Let us consider a two-dimensional problem of solid mechanics in domain $\Omega$ bounded by $\Gamma$ whose strong-form of governing equation and the essential boundary conditions are given by:

$$
\begin{gathered}
\sigma_{\mathrm{ij}, \mathrm{j}}(\mathrm{x})+\mathrm{b}_{\mathrm{i}}(\mathrm{x})=\mathrm{0} \\
\sigma_{\mathrm{ij}} \mathrm{n}_{\mathrm{j}}=\mathrm{t}_{\mathrm{i}}^{0} \text { on } \Gamma_{\mathrm{t}} \\
\mathrm{u}_{\mathrm{i}}=\mathrm{u}_{\mathrm{i}}^{0} \text { on } \Gamma_{\mathrm{u}}
\end{gathered}
$$


A Comparative Study of Size Parameters Effects in Meshless Method Local Petrov-Galerkin (Mlpg) and Local Radial Point Interpolation Method (Lrpim)

Where in $\Omega: \boldsymbol{\sigma}^{\mathrm{T}}=\left[\sigma_{\mathrm{xx}}, \sigma_{\mathrm{yy}}, \tau_{\mathrm{xy}}\right]$ is the stress vector, $\mathbf{b}^{\mathrm{T}}=\left[\mathrm{b}_{\mathrm{x}}, \mathrm{b}_{\mathrm{y}}\right]$ the body force vector. $\mathbf{n}=\left(\mathrm{n}_{1}, \mathrm{n}_{2}\right)$ denotes the vector of unit outward normal at a point on the natural boundaries

$\mathrm{t}^{0}$ is the prescribed effort, $\left[\mathrm{u}_{1}, \mathrm{u}_{2}\right]$ the displacement components in the plan and $\left[\mathrm{u}_{1}^{0}, \mathrm{u}_{2}^{0}\right]$ on the essential boundaries. In the local Petrov-Galerkin approaches [1] one may write a weak form over $\Omega_{\mathrm{Q}}$ a local quadrature domain (for node I), which may have an arbitrary shape, and contain the point $x_{Q}$ in question, see (Fig. 1). The generalized local weak form of the differential eq. (7) is obtained by:

$$
\int_{\Omega_{\mathrm{Q}}}\left(\sigma_{\mathrm{ij}, \mathrm{j}}(\mathrm{x})+\mathrm{b}_{\mathrm{i}}(\mathrm{x})\right) v_{\mathrm{I}} \mathrm{d} \Omega=0
$$

Where $\Omega_{\mathrm{Q}}$ is the local domain of quadrature for node I and $v_{\mathrm{I}}$ is the weight or test function,

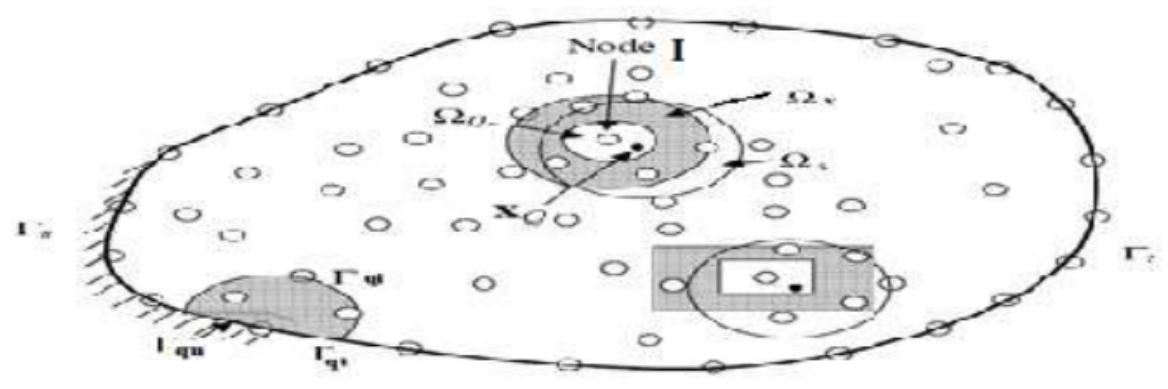

Figure 1 The local sub-domains around point $\mathrm{x}_{\mathrm{Q}}$ and boundaries

Using the divergence theorem [2] in eq.(10), we obtain: $\int_{\Gamma_{Q}} \sigma_{\mathrm{ij}} \mathrm{n}_{\mathrm{j}} \mathrm{v}_{\mathrm{l}} \mathrm{d} \Gamma-\int_{\Omega_{Q}} \sigma_{\mathrm{ij}} v_{1, \mathrm{j}} \mathrm{d} \Omega+\int_{\Omega_{Q}} \mathrm{~b}_{\mathrm{i}} v_{\mathrm{I}} \mathrm{d} \Omega=0$ (11)

Where $\Gamma_{\mathrm{Q}}=\Gamma_{\mathrm{Qi}} \cup \Gamma_{\mathrm{Qu}} \cup \Gamma_{\mathrm{Qt}} ; \Gamma_{\mathrm{Qi}}$ : The internal boundary of the quadrature domain; $\Gamma_{\mathrm{Qt}}$ : The part of the natural boundary that intersects with the quadrature domain; $\Gamma_{\mathrm{Qu}}$ : The part of the essential boundary that intersects with the quadrature domain

$$
\begin{aligned}
& \text { We can then change the expression of eq (11): } \\
& \int_{\Gamma_{\mathrm{Qi}}} \sigma_{\mathrm{ij}} \mathrm{n}_{\mathrm{j}} v_{\mathrm{I}} \mathrm{d} \Gamma+\int_{\Gamma_{\mathrm{Qu}}} \sigma_{\mathrm{ij}} \mathrm{n}_{\mathrm{j}} v_{\mathrm{I}} \mathrm{d} \Gamma+\int_{\Gamma_{\mathrm{Q}}} \sigma_{\mathrm{ij}} \mathrm{n}_{\mathrm{j}} v_{\mathrm{I}} \mathrm{d} \Gamma-\int_{\Omega_{\mathrm{Q}}} \sigma_{\mathrm{ij}} v_{\mathrm{I}, \mathrm{j}} \mathrm{d} \Omega+\int_{\Omega_{\mathrm{Q}}} \mathrm{b}_{\mathrm{i}} v_{\mathrm{I}} \mathrm{d} \Omega=0
\end{aligned}
$$

Using the RPIM shape functions (see sub-section 2), we can approximate the trial function for the displacement at a point as eq (6) the stress vector is defined by:

$\boldsymbol{\sigma}=\mathrm{C} \boldsymbol{\varepsilon}=\mathrm{CL}_{\mathrm{d}} \mathrm{u}^{\mathrm{h}}$

Where $\mathbf{C}$ is the symmetric elasticity tensor of the material

$$
\mathbf{C}=\left(\begin{array}{ccc}
E /\left(1-v^{2}\right) & v E /\left(1-v^{2}\right) & 0 \\
v E /\left(1-v^{2}\right) & E /\left(1-v^{2}\right) & 0 \\
0 & 0 & E / 2(1+v)
\end{array}\right)
$$

Equation (12) can be written:

$$
\int_{\Omega_{\mathrm{Q}}} \overline{\mathrm{V}}_{\mathrm{I}}^{\mathrm{T}} \boldsymbol{\alpha} \Omega-\int_{\Gamma_{\mathrm{Qi}}} \mathrm{tV} \mathrm{d} \Gamma-\int_{\Gamma_{\mathrm{Qu}}} \mathrm{t} \mathrm{V}_{\mathrm{l}} \mathrm{d} \Gamma=\int_{\Gamma_{\mathrm{Qi}}} \mathrm{t}^{0} \mathrm{~V}_{\mathrm{I}} \mathrm{d} \Gamma+\int_{\Omega_{\mathrm{Q}}} \mathrm{V}_{\mathrm{I}} \mathrm{bd} \Omega
$$


Where $\overline{\mathbf{V}}_{I}=\left(\begin{array}{cc}v_{I, x} & 0 \\ 0 & v_{y, y} \\ v_{y, y} & v_{I, x}\end{array}\right)$ is a matrix that contains the derivatives of the weight functions and $\mathbf{V}=\left(\begin{array}{cc}v_{\mathrm{I}} & 0 \\ 0 & v_{\mathrm{I}}\end{array}\right)$ is the matrix of weight function. ; Substituting the differential operator $\mathbf{L}_{\mathbf{d}}=\left(\begin{array}{cc}\partial / \partial \mathbf{x} & 0 \\ 0 & \partial / \partial \mathrm{y} \\ \partial / \partial \mathrm{y} & \partial / \partial \mathrm{x}\end{array}\right)$ into $(13)$

We obtain: $\boldsymbol{\sigma}=\mathbf{C} \sum_{\mathrm{I}=1}^{\mathrm{n}_{\mathrm{t}}} \mathbf{B}_{\mathrm{I}} \mathbf{u}_{\mathrm{I}}$

Where ${ }_{\mathbf{B}_{\mathrm{I}}}=\left(\begin{array}{cc}\Phi_{\mathrm{I}, \mathrm{x}} & 0 \\ 0 & \Phi_{\mathrm{I}, \mathrm{y}} \\ \Phi_{\mathrm{I}, \mathrm{y}} & \Phi_{\mathrm{I}, \mathrm{x}}\end{array}\right)$. By using the matrix $\mathbf{L}_{\mathrm{n}}=\left(\begin{array}{cc}\mathrm{n}_{1} & 0 \\ 0 & \mathrm{n}_{2} \\ \mathrm{n}_{2} & \mathrm{n}_{1}\end{array}\right)$, the tractions of a point $\mathrm{x}$ can be written as:

$$
\mathbf{t}=\mathbf{L}_{\mathrm{n}}^{\mathrm{T}} \boldsymbol{\sigma}
$$

Substituting equations $(15,16)$ into equation $(14)$, we obtain the discrete systems of linear equations for the node I.

$\sum_{\mathrm{I}=1}^{\mathrm{n}_{\mathrm{t}}}\left[\int_{\Omega_{\mathrm{Q}}} \overline{\mathbf{V}}_{\mathrm{I}}^{\mathrm{T}} \mathbf{C B} \mathrm{d} \Omega-\int_{\Gamma_{\mathrm{Qi}}} \mathrm{L}_{\mathrm{h}}^{\mathrm{T}} \mathbf{C B} \mathbf{V}_{\mathrm{I}} \mathrm{d} \Gamma-\int_{\Gamma_{\mathrm{Qu}}} \mathrm{L}_{\mathrm{n}}^{\mathrm{T}} \mathrm{CB}_{\mathrm{I}} \mathbf{V}_{\mathrm{I}} \mathrm{d} \Gamma\right] \mathbf{u}_{\mathrm{l}}=\int_{\Gamma_{\Gamma_{\mathrm{Q}}}} \mathrm{t}^{0} \mathbf{V}_{\mathrm{I}} \mathrm{d} \Gamma+\int_{\Omega_{\mathrm{Q}}} \mathrm{V}_{\mathrm{I}} \mathbf{b} \mathrm{d} \Omega$

The matrix form of Eq.(17) can be written as in matrix form:

$\sum_{\mathrm{I}=1}^{\mathrm{n}_{\mathrm{t}}} \mathbf{K}_{\mathrm{I}} \mathbf{u}_{\mathrm{I}}=\mathbf{f}_{\mathrm{I}}$

Where expression of nodal matrix

$\mathrm{K}_{\mathbf{I}}$ Is: $\mathbf{K}_{\mathbf{I}}=\int_{\Omega_{\mathrm{Q}}} \overline{\mathbf{V}}_{\mathrm{I}}^{\mathrm{T}} \mathrm{CB}_{\mathrm{I}} \mathrm{d} \Omega-\int_{\Gamma_{\mathrm{Qi}}} \mathrm{L}_{\mathrm{n}}^{\mathrm{T}} \mathrm{CB}_{\mathrm{I}} \mathrm{V}_{\mathrm{I}} \mathrm{d} \Gamma-\int_{\Gamma_{\mathrm{Qu}}} \mathrm{L}_{\mathrm{n}}^{\mathrm{T}} \mathrm{CB}_{\mathrm{I}} \mathrm{V}_{\mathrm{I}} \mathrm{d} \Gamma$

And nodal force vector with contributions from body forces applied in the problem domains

$$
\mathbf{f}_{\mathrm{I}}=\int_{\Gamma_{\mathrm{Qt}}} \mathrm{t}^{0} \mathrm{~V}_{\mathrm{I}} \mathrm{d} \Gamma+\int_{\Omega_{\mathrm{Q}}} \mathrm{V}_{\mathrm{I}} \mathbf{b} \mathrm{d} \Omega
$$

Where $n_{0}$ denote the set of the nodes in the support domain $\Omega_{\mathrm{S}}$ of point $\mathrm{x}_{\mathrm{Q}}$.

Two independent linear Eqs $(19,20)$ can be obtained for each node in the entire problem domain and by assembling all these $2 * n$ equations to obtain the final global system equations:

$\mathbf{k}_{2 \mathrm{n} * 2 \mathrm{n}} \mathbf{u}_{2 \mathrm{n} * 1}=\mathbf{f}_{2 \mathrm{n} * 1}$

To solve the precedent system, the standard Gauss quadrature formula is applied with 16 Gauss points [9] for calculating integrals in Eqs $(19,20)$ on both boundary and domain.

\section{NUMERICAL EXAMPLE FOR 2D ELASTOSTATIC}

This section is about numerical results for a cantilever rectangular plate see (Fig. 2a). First, were investigated the effect of the size of support domain and was examined numerically convergence of two methods LRPIM and MLPG for several materials; then, comparisons will 
be made with the analytic solution for several materials [10]. \{We choose: steel, zinc, aluminum and copper with: $E=3.10^{7} \mathrm{~N} / \mathrm{m}^{2}, v=0.3 ; \mathrm{E}=113.10^{5} \mathrm{~N} / \mathrm{m}^{2}, v=0.25, \mathrm{E}=1.10^{7} \mathrm{~N} / \mathrm{m}^{2}$ ,$v=0.34 ; \mathrm{E}=17.10^{6} \mathrm{~N} / \mathrm{m}^{2}, v=0.33$ respectively\} Dimension of the plate are denoted: height $\mathrm{D}=12 \mathrm{~m}$, length $\mathrm{L}=48 \mathrm{~m}$, the thickness: unit and finally for Loading: $\mathrm{P}=10^{3} \mathrm{~N}$.
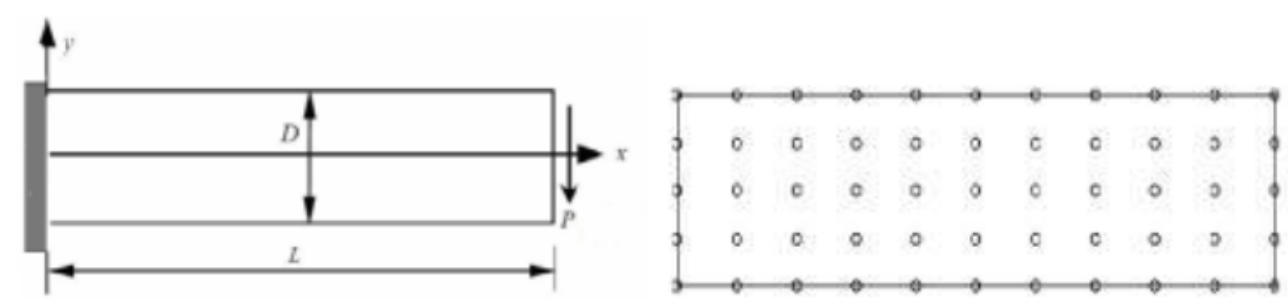

Figure 2 a-b. Plate subjected to distributed traction at the free end and regular field nodes distribution on the problem domain and boundaries

In our numerical calculations, were considered many regular distributions of nodes $\mathbf{n}_{\mathrm{t}}: 55$, 91, 175 and 189. To calculate the error energy, a background cells are required; then, for each value of $\mathbf{n}_{\mathbf{t}}$ the number of cell was varied. To obtain numerical values, the distribution of the deflection through the plates, size of support domain is varied and $\alpha_{Q}=2$. The sizes of support domain $\Omega_{\mathbf{s}}$ (quadrature domain $\Omega_{Q}$ resp.) are defined by: $\mathrm{d}_{\mathrm{s}}=\alpha_{\mathrm{s}} \mathrm{d}_{\mathrm{c}}$ (see Fig. $2 \mathrm{~b}$ ).

\section{RESULTS AND DISCUSSIONS}

\subsection{Effect of spline functions and the number of field nodes.}

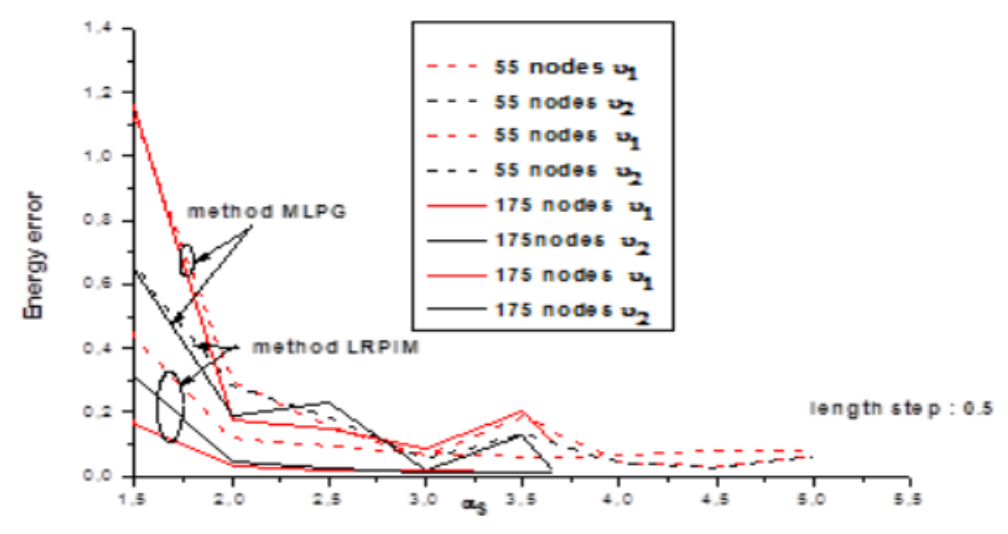

Figure 3 Variation of the energy error as a function of $\alpha_{\mathrm{S}}$ for two spline functions, MLPG and

$$
\text { LRPIM methods }\left(\mathbf{n}_{\mathbf{t}}=55,175 \text { and steel }\right)
$$

Figure 3 shows the energy error as a function of $\alpha_{\mathrm{S}}$ for two spline functions (cubic $\left(v_{1}\right)$ and quartic $\left.\left(v_{2}\right)\right)$ and the number of field nodes $\mathbf{n}_{\mathbf{t}}=55,175$. We chose a steel plate with results of both methods MLPG and LRPIM. The latter has the radial basis multi-quadrics (RBF-MQ) function. We notice that if the values $\alpha_{S}$ are less than 1.80, the error energy is great for both methods MLPG and LRPIM; therefore, the methods are not convergent. Furthermore, we can see that there is an oscillation between the values $\alpha_{\mathrm{S}}\left(2<\alpha_{\mathrm{s}}<4\right)$ concerning MLPG method. 
Moreover, from $\alpha_{\mathrm{S}}=4$, we note that both methods LRPIM and MLPG are well performed. The curves have the same shape in each method, so the LRPIM method has a good

Convergence starting from $\alpha_{\mathrm{S}}=1.80$. The domain of convergence has its upper end to $\alpha_{\mathrm{S}}=5$ for $\mathbf{n}_{\mathbf{t}}=55$. Concerning $\mathbf{n}_{\mathbf{t}}=175$ the maximum value $\alpha_{\mathrm{s}}$ decreases and takes the value of $\alpha_{\mathrm{S}}=3.66[11]$.

\subsection{Effect of the radial basis functions RBF (multi-quadrics (MQ) function, the Gaussian (Exp) function and the thin plate spline (TPS) function}
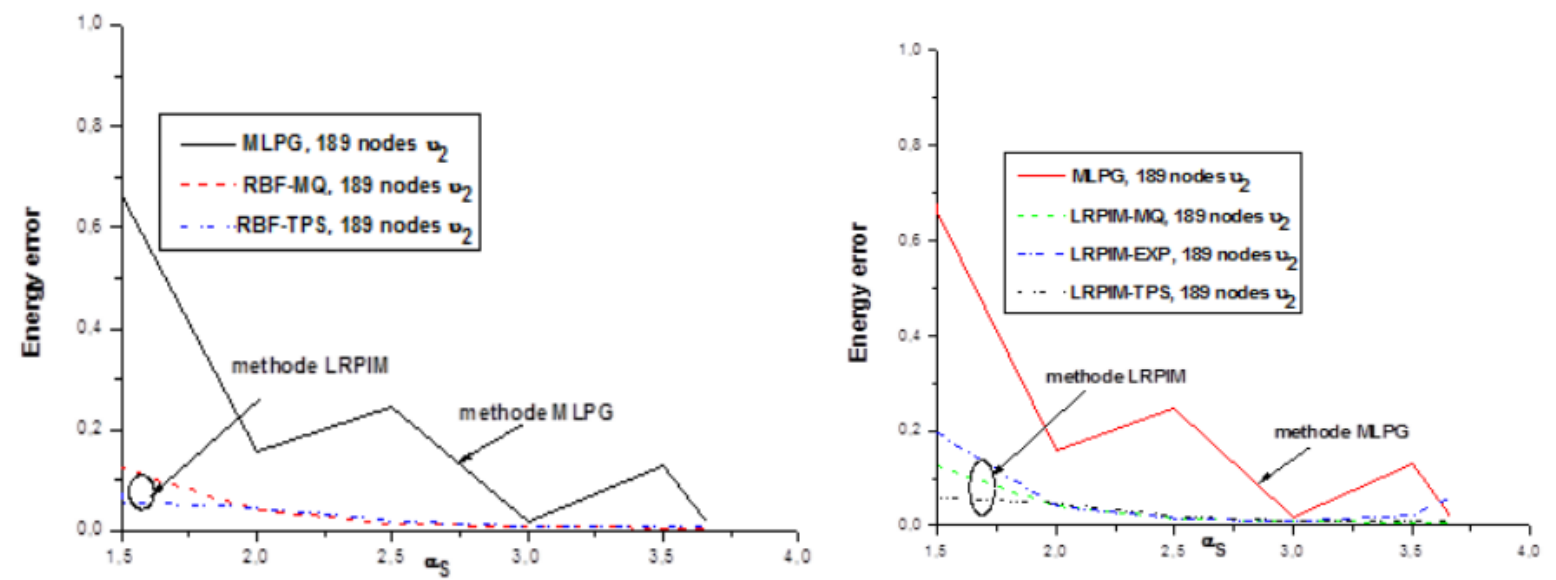

Figure 4-5 Variation of the energy error as a function of $\alpha_{\mathrm{S}}$ for two bases RBF (MQ, EXP and TPS) for MLPG and LRPIM methods $\left(\mathbf{n}_{\mathbf{t}}=189\right.$ and $\left.v_{2}\right)$.

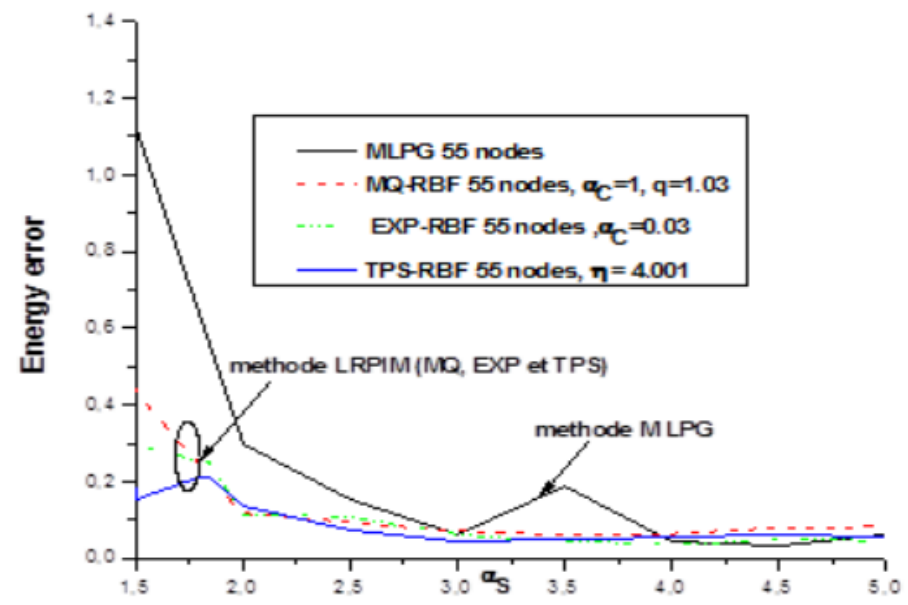

Figure 6 Variation of the energy error as a function of $\alpha_{\mathrm{S}}$ for different bases RBF (MQ, EXP and TPS) and $v_{1}$ for MLPG and LRPIM methods $\left(\mathbf{n}_{\mathbf{t}}=55\right.$ and $\left.v_{1}\right)$.

Figures 4-6 illustrate the variations of the energy error as a function of $\alpha_{\mathrm{S}}$ with different radial basis functions of LRPIM method and the number of field nodes $\mathbf{n}_{\mathbf{t}}=55,189$. We chose the optimal shape parameters: ( $\mathrm{q}=1.03$ and $\left.\alpha_{\mathrm{C}}=4\right)$ for RBF-MQ, $\left(\alpha_{\mathrm{C}}=0.03\right.$ ) for RBFEXP and ( $\eta=4.001)$ for RBF-TPS. The LRPIM method with different radial basis functions gives a lower energy starting from $\alpha_{\mathrm{S}}=1.80$ [12]. It is remarked that there is no dependence 
between the extremities of the domain of convergence. If $\mathbf{n}_{\mathbf{t}}=55, \alpha_{\mathrm{S}}=5$ and if $\mathbf{n}_{\mathbf{t}}=189$, the maximum value of $\alpha_{\mathrm{S}}$ decreases and takes the value $\alpha_{\mathrm{S}}=3.66$. The results obtained [11] for the studied spline functions are consistent with those found by the MLPG method. The results found by MLPG are very good.

\subsection{Effect of different materials.}
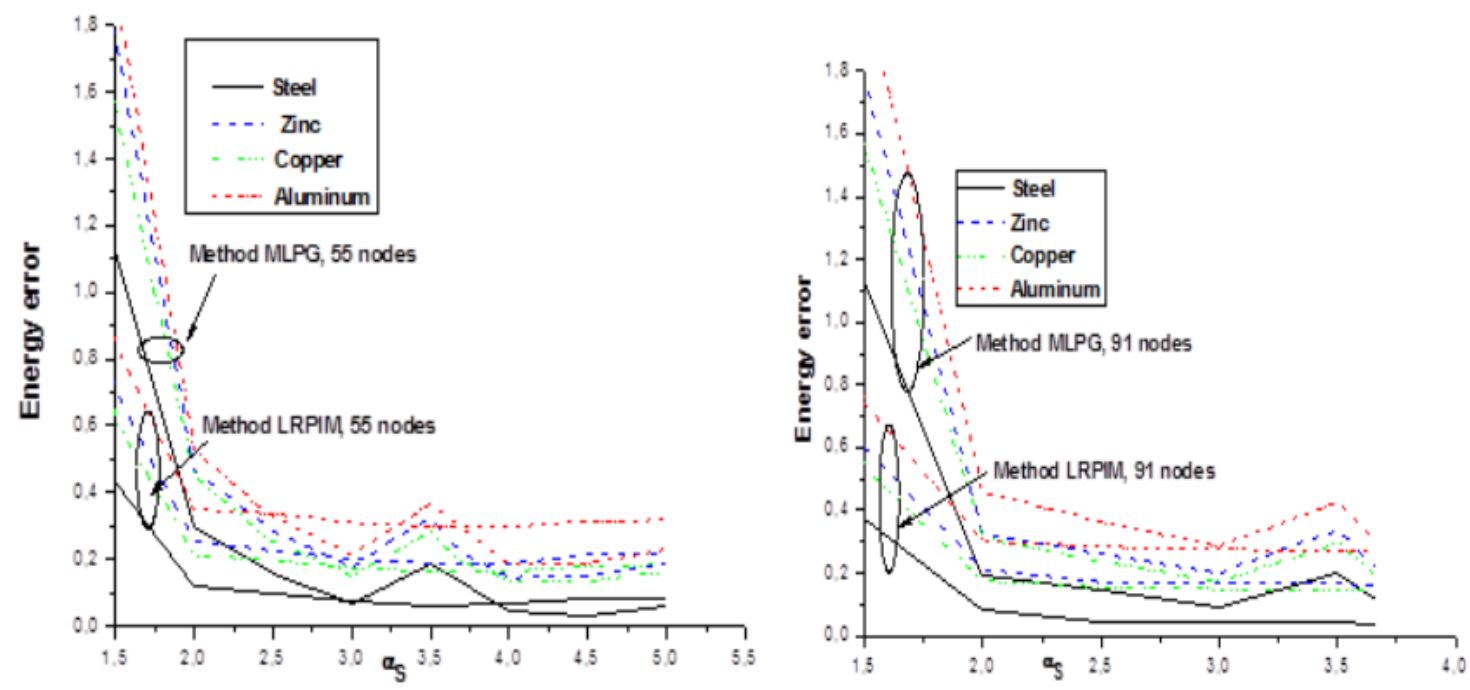

Figure 7-8 Variation of the energy error as a function of $\alpha_{\mathrm{S}}$ for different materials and $v_{1}$ for MLPG and LRPIM methods $\left(\mathbf{n}_{\mathbf{t}}=55,91\right)$

Figures 7-8 display the variation of energy error as a function of $\alpha_{\mathrm{s}}$ for different materials, for the number of field nodes $\mathbf{n}_{\mathbf{t}}=55,91$ and for the cubic spline function of both methods MLPG and LRPIM. It is clear that there is an oscillation between the values of $\alpha_{\mathrm{S}}: 2<\alpha_{\mathrm{S}}<4$ (Fig.7) and between the values of $\alpha_{\mathrm{S}}: 2<\alpha_{\mathrm{S}}<3.66$ (Fig.8) for MLPG method. For LRPIM method with different values of $\mathrm{E}$ and $v$ ie different rectangular plates (steel, zinc, copper and aluminum), starting from $\alpha_{\mathrm{s}}=1.80$, so the method LRPIM is convergent. When $\alpha_{\mathrm{S}}$ is between the values 4 and 5 (Fig.7), both methods MLPG and LRPIM show a good convergence. We note that all the curves of each of the different materials studied have the same shape for a fixed value of $\alpha_{\mathrm{S}}$. The obtained results are compared with the results found by method MLPG [11] for different materials.

\subsection{Effect of number of nodes}

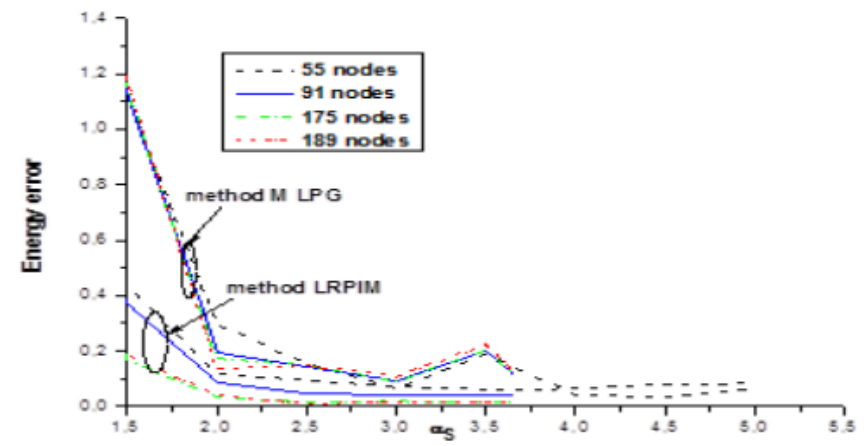


Figure 9 Variation of the energy error as a function of $\alpha_{\mathrm{S}}$ for different number of field nodesn $\boldsymbol{n}_{\mathbf{t}}$ and $v_{1}$ for MLPG and LRPIM.

Figure 9 displays the variation of energy error as a function of $\alpha_{\mathrm{s}}$ for different number of field nodes $\mathbf{n}_{\mathbf{t}}=55,91,175,189$ and the cubic spline function $\left(v_{1}\right)$ of both methods MLPG and LRPIM. It is crucial to notice that if the values $\alpha_{\mathrm{S}}$ are less than 1.80 , the error energy is great with the oscillation between the values of $\alpha_{\mathrm{S}}: 2<\alpha_{\mathrm{S}}<4$ for $\mathbf{n}_{\mathrm{t}}=55$ and between the values of $\alpha_{\mathrm{S}}$ $: 2<\alpha_{\mathrm{S}}<3.66$ for other values of node numbers $\mathbf{n}_{\mathbf{t}}$ in the method MLPG. For LRPIM method with different values of $\mathbf{n}_{\mathbf{t}}$ number of nodes, the energy error is low for steel starting from $\alpha_{\mathrm{S}}$ $=1.80$. Thus, the LRPIM method is convergent. When $\alpha_{\mathrm{S}}$ is between 4 and 5 , both methods MLPG and LRPIM have good convergence for $\mathbf{n}_{\mathbf{t}}=55$. The results are identical, so the effect of $\mathbf{n}_{\mathbf{t}}$ is almost the same for the both methods.

\section{CONCLUSION}

The convergence and the accuracy of both methods MLPG and LRPIM are dependent on the size of support domain, number of nodes, spline functions, radial basis function RBF (MQ, EXP and TPS) for LRPIM method. The choice number of nodes that has its upper end to $\alpha_{\mathrm{S}}=5$ for $\mathbf{n}_{\mathbf{t}}=55$, and for $\mathbf{n}_{\mathrm{t}}=(91,175,189)$ the maximum value of $\alpha_{\mathrm{s}}$ decreases and takes the value $\alpha_{\mathrm{S}}=3.66$. These results are in agreement with the analytical solution. The domain of convergence is independent of the choice of the studied materials: steel, zinc, copper and aluminum, with values $\mathrm{n}_{\mathrm{t}}=55, \alpha_{\mathrm{S}}=5$ and $n_{t}=91,175$ and $189 \alpha_{\mathrm{S}}=3.66$. Finally, this study showed that the domain of convergence is the same for both methods LRPIM and MLPG. No dependence between the ends of the domain of convergence is noticed. If $\mathbf{n}_{\mathbf{t}}=55$ then $\alpha_{\mathrm{S}}=5$ is maximum and if $\mathbf{n}_{\mathbf{t}}=\left(91,175\right.$ and 189), so this value decreases to $\alpha_{\mathrm{S}}=3.66[11]$.

\section{REFERENCE}

[1] T. Belyschko, YY. Lu, L. Gu, Element - free Galerkin methods. Int J Numer Meth Eng. 37(1994) 229-256.

[2] S. N. Atluri, T. Zhu, A new meshless local Petrov-Galerkin (MLPG) approach in computational mechanics, Comput Mech. 22(1998)117-127.

[3] S. N. Atluri, S. Shen, The meshless local Petrov-Galerkin (MLPG) method, Tech Science Press, Forsyth. (2002).

[4] G. R. Liu, Y. T. Gu, A local point interpolation method for stress analysis of twodimensional solids, Struct Eng Mech. 11(2) (2001a) 221-236.

[5] G. R. Liu, Y. T. Gu, A point interpolation method for two dimensional solids, Int J Numer Methods Eng. 50(2001c) 937-951.

[6] G. Wang and G.R. Liu, on the optimal shape parameters of radial basis functions used for 2-D meshless methods, Comput Methods Appl, Mech. Engrg. 191(2006) 2611-2630.

[7] G. R. Liu, L. Yan, J. G. Wang, Y. T. Gu, Point interpolation method based on local residual formulation using radial basis functions. Struct Eng Mech. 14(6)(2002)713-732.

[8] M.A. Golberg, C.S. Chen, H. Bowman, Some recent results and proposals for the use of radial basis functions in the BEM, Engineering Analysis with Boundary Elements. 23(1999) 285-296. 
A Comparative Study of Size Parameters Effects in Meshless Method Local Petrov-Galerkin (Mlpg) and Local Radial Point Interpolation Method (Lrpim)

[9] A. Quarteroni, R. Sacco, F. Saleri, Méthodes Numériques, Algorithmes, analyse et applications, Springer.(2007).

[10] S. P. Timoshenko and J. N. Goodier, Theory of Elasticity, rd 3 edition, McGraw Hill. (1970).

[11] A. Moussaoui and T. Bouziane, Convergence of MLPG Method for Various Materials of a 2D Problem, Applied Mathematical Sciences. 8 (69) (2014)3405 - 3418.

[12] Chandram Karri, Sudhakar AVV, Invasive Weed Optimization for Optimal Capacitor Placement on a Radial Distribution System. International Journal of Electrical Engineering \& Technology, 8(5), 2017, pp. 9-19.

[13] A. Moussaoui and T. Bouziane, Numerical study of the shape parameter dependence of the local radial point interpolation method in linear elasticity, Journal MethodsX.3(2016)178187.

[14] Dr.K.Ravichandrudu, G.Jyothi, Mr.P.Yohan Babu and, Mr.G.V.P.Anjaneyulu, Artificial Bee Colony Algorithm Based Approach for Capacitor Allocation in Unbalanced Radial Distribution Systems, International Journal of Electrical Engineering and Technology (IJEET), Volume 4, Issue 4, July-August (2013), pp. 67-73 